Old Dominion University

ODU Digital Commons

VMASC Publications

Virginia Modeling, Analysis \& Simulation Center

2014

\title{
Toward a Formalism of Modeling and Simulation Using Model Theory
}

\author{
Saikou Y. Diallo \\ Old Dominion University, sdiallo@odu.edu \\ Jose Padilla \\ Old Dominion University, jpadilla@odu.edu \\ Ross Gore \\ Old Dominion University, rgore@odu.edu \\ Heber Herencia-Zapana \\ Andreas Tolk \\ Old Dominion University, atolk@odu.edu
}

Follow this and additional works at: https://digitalcommons.odu.edu/vmasc_pubs

Part of the Engineering Commons, $\underline{\text { Mathematics Commons, and the Science and Technology }}$ Studies Commons

\section{Repository Citation}

Diallo, Saikou Y.; Padilla, Jose; Gore, Ross; Herencia-Zapana, Heber; and Tolk, Andreas, "Toward a Formalism of Modeling and Simulation Using Model Theory" (2014). VMASC Publications. 12.

https://digitalcommons.odu.edu/vmasc_pubs/12

\section{Original Publication Citation}

Diallo, S. Y., Padilla, J. J., Gore, R., Herencia-Zapana, H., \& Tolk, A. (2014). Toward a formalism of modeling and simulation using model theory. Complexity, 19(3), 56-63. doi:10.1002/cplx.21478

This Article is brought to you for free and open access by the Virginia Modeling, Analysis \& Simulation Center at ODU Digital Commons. It has been accepted for inclusion in VMASC Publications by an authorized administrator of ODU Digital Commons. For more information, please contact digitalcommons@odu.edu. 


\title{
Toward a Formalism of Modeling and Simulation Using Model Theory
}

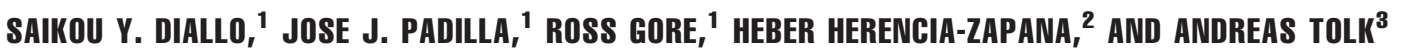 \\ ${ }^{1}$ Virginia Modeling Analysis and Simulation Center, Old Dominion University 1030 University Blvd, \\ Suffolk, Virginia 23435; ${ }^{2}$ National Institute of Aerospace, Hampton, Virginia; and ${ }^{3}$ Chief Scientist Simis \\ Inc., Portsmouth, Virginia
}

Received 22 April 2013; accepted 17 September 2013

\begin{abstract}
This article proposes a Modeling and Simulation (M\&S) formalism using Model Theory. The article departs from the premise that M\&S is the science that studies the nature of truth using models and simulations. Truth in models and simulations is relative as they seek to answer specific modeling questions. Consequently, truth in M\&S is relative because every model is a purposeful abstraction of reality. We use Model Theory to express the proposed formalism because it is built from the premise that truth is relative. The proposed formalism allows us to: (1) deduce formal definitions and explanations of areas of study in M\&S, including conceptual modeling, validity, and interoperability, and (2) gain insight into which tools can be used to semi-automate validation and interoperation processes. (C) 2013 Wiley Periodicals, Inc. Complexity 19: 56-63, 2014
\end{abstract}

Key Words: modeling and simulation; verification \& validation

\section{INTRODUCTION}

$\mathrm{F}$ ormalisms of Modeling and Simulation (M\&S) have traditionally taken either a Systems Engineering approach [1] or a Computer Science approach [2] both focusing with formalizing the simulation aspects of M\&S. Similarly, attempts to study the complexity of M\&S have also come from systems science [3] and resulted in levels of complexity for M\&S as it applies to modeling complex systems. However, while the current focus on simulation has provided great advances in terms of com-

Correspondence to: Saikou Y. Diallo, Virginia, Modelling, Analysis and Simulation Center, Old Dominion University, VA 2343.E-mail: sdiallo@odu.edu putability of models, advances in modeling have been convoluted in the simulation activity. We argue that the atomicity of M\&S is what makes it a scientific discipline. Modeling without simulation is systems engineering and simulation without modeling is software engineering. This is specially true when dealing with problem situations which are problems whose specification is not universally agreed upon [4].

Most engineering disciplines assume a problem and its solution space are well defined. As an engineering discipline, $M \& S$ is used to solve well-defined problems with available algorithms and generate solutions that can be empirically validated. This is the case, for example, of physics-based models where the objective is to replicate the behavior of a physical system. $M \& S$ is also used to 
gain insight through the identification of relevant parameters and the exploration of possible relations between entities [5]. Both uses of M\&S are special cases of dealing with problem situations in that they either depart from a well-defined problem or attempt to define the problem through the exploration of a solution space. In general, M\&S problems are characterized by (1) the lack of consensus on the existence of a problem and (2) where there is consensus, by the lack of a unique universally agreed upon specification of the problem. Consequently, M\&S solutions are merely explanations of a situation based on a given understanding of that situation. Explanations are the result of different perspectives of the same problem situation which leads to potentially different truth values. This makes M\&S valuable in the study of complex systems in general and social sciences in particular $[6,7]$.

Recently, there have been discussions on the complexification of engineering. These discussions have created an emerging trend of equating engineering with science [8]. Since M\&S deals with problem situations it is a science of trade offs and compromise. It is a trade-off between reality and a model thereof and a trade-off between computability and simulatibility. Here we use the term simulatibility to mean the ability to simulate a model with a mix of live, virtual, and constructive means. It is a compromise between an evaluation of a simplification of reality and reality itself. M\&S studies what models and simulations capture, how truthful models and simulations are, and how to compose models and simulations. These three areas of study correspond to conceptual modeling, validity, and interoperability, respectively.

According to Robinson [9], conceptual modeling is perhaps the most important aspect of simulation modeling and the least understood. Robinson provides a descriptive definition of conceptual model (p. 65): "The conceptual model is a non-software specific description of the simulation model that is to be developed, describing objectives, inputs, outputs, content, assumptions, and simplifications of the model." Robinson notes the importance of separating the conceptual model from the software implementation and the components of a conceptual model. In this article, we provide a formal definition of the term conceptual model. This definition complements the discussion of the role of conceptual modeling in M\&S found in [10].

Validity has been extensively studied in not only M\&S but also in engineering, natural sciences, and philosophy. However, validity has been mostly discussed as empirical validity. Empirical validity can be established in M\&S when comparing the results of a simulation with observations of a well-bounded phenomenon or system. Yet, this is not always possible under problem situations. The article provides a formal definition of axiomatic validity by tying together the concepts of reference model, conceptual model, and simulation.
Finally, interoperability is understood as "the ability of two or more systems or components to exchange information and to use the information that has been exchanged" [11]. This informal view of interoperability is widely reflected in the literature. However, this view is ambiguous on the meaning of the terms "exchange," "information" and "use." The article provides a formal definition of interoperability by deducing that validity is a special form of interoperability.

In order to capture the definitions of conceptual model, validity, and interoperability, we use Model Theory. Model Theory is a branch of mathematics that studies the relationships among objects, structures, and the nature of truth [12]. It provides the grounds for formal definitions and serves as a deductive means for arriving to consistent models and simulations.

The remainder of this article proceeds as follows. First, we present a brief introduction to Model Theory to establish the formalism we employ to specify M\&S. Then, we explore the implications of our $M \& S$ specification in the areas of conceptual modeling, validity, and interoperability. Finally, we summarize our contributions.

\section{MODEL THEORY-BASED M\&S FORMALISM (MT-MS FORMALISM) \\ Introduction to Model Theory}

Model Theory sits at the intersection of mathematics, philosophy, and computer science. It focuses on the syntactic and semantic study of structures including formal languages, group fields, or graphs. Given its interdisciplinary roots, purpose, and openness to the premise of a relative truth [12], it has the ability to combine both the modeling and simulation aspects of M\&S. In related work it has been proposed as a way to formally describe $M \& S$ and has been used to formally define key terms to derive a framework for developing M\&S systems [10]. Here we focus on the formal representation of $M \& S$ in order to elucidate its basic properties and provide insight into the discipline.

We use basic concepts of Model Theory and purposefully stay away from more complex notion such as sorts or fields. The interested reader is encouraged to consult [12].

\section{Definition 1}

A language $\mathscr{2}$ is a set consisting of all logical symbols with perhaps some constant, function and/or relational symbols included.

\section{Definition 2}

A model (or structure) $\mathcal{U}$ for a language $\mathscr{Q}$ is an ordered pair $\mathcal{U}=\left\langle A,\left\{R_{n}\right\}_{n \geq 1}\right\rangle$, where $A$ is a set and $R_{n}$ is a relation such that $\left(a_{1}, a_{2}, \cdots, a_{n}\right) \in R_{n}$ if and only if there exists an 
$f$ such that $f\left(a_{1}, a_{2}, \cdots, a_{n-1}\right)=a_{n}$. A sentence is an assertion that can be assigned the Boolean value of true or false. Let the set of elementary sentences $L_{e}=\left\{\varphi_{j}\right\}$. They are called elementary because using the logical operators and, or, negation, etc., the language $\mathscr{Q}$ is generated

$$
L=\left\{\varphi: \varphi \text { is generated by elements of } L_{e}\right\}
$$

As stated in Ref. [12], the most fundamental concept is that of a sentence $\sigma$ being true when interpreted in a model $\mathcal{U}$ which can be written as $\mathcal{U} \vDash \sigma$. This concept is extended in the following definitions.

\section{Definition 3}

If $\Sigma$ is a set of sentences, $\mathcal{U}$ is said to be a model of $\Sigma$, written $\mathcal{U} \vDash \Sigma$, whenever $\mathcal{U} \vDash \sigma$ for each $\sigma \in \Sigma$. $\Sigma$ is said to be satisfiable iff there is some $\mathcal{U}$ such that $\mathcal{U} \vDash \Sigma$.

\section{Definition 4}

A theory $\mathcal{T}$ is a set of sentences. If $\mathcal{T}$ is a theory and $\sigma$ is a sentence, we write $\mathcal{T} \vDash \sigma$ whenever we have that for all $\mathcal{U}$ if $\mathcal{U} \vDash \mathcal{T}$ then $\mathcal{U} \vDash \sigma$. We say that $\sigma$ is a consequence of $\mathcal{T}$. A theory is said to be closed whenever it contains all consequences.

\section{Definition 5}

If $\mathcal{U}$ is a model of $\mathscr{Q}$, the theory of $\mathcal{U}$, denoted $\mathrm{Th} \mathcal{U}$, is defined to be set of all sentences of $\mathscr{Q}$ which are true in $\mathcal{U}$,

$$
\{\sigma \text { of } \mathscr{Q}: \mathcal{U} \vDash \sigma\}
$$

\section{Definition 6}

$\Sigma \subseteq \mathcal{T}$ is said to be a set of axioms for $\mathcal{T}$ whenever $\Sigma \models \sigma$ for every $\sigma$ in $\mathcal{T}$; in this case: $\Sigma \models \mathcal{T}$.

\section{Definition 7}

A finite state machine is a triple $\{I, S, O\}$ where $I$ is the set of inputs, $S$ is the set of states, and $\mathrm{O}$ is the set of outputs.

The string $s=s_{0} s_{1} s_{2} \ldots s_{m}$ is the $m$ realization of the finite state machine. Now the definition of a language generated by the finite state machine is given.

\section{Definition 8}

A language $\mathscr{2}_{\mathrm{FSM}}$ is a mapping, $\mathscr{2}$, from $S$ to the set of atomic sentences $\left\{\varphi_{i}: i=1, \cdots, m\right\}$.

It is important to note that definitions 1 and 2 state that a language $\mathscr{Q}$ can be built from a structure $\mathcal{U}$. In other words, sentences can be built using $A$ and $R$. On the other hand, Definition 5 states that from the language $\mathscr{2}$ one can identify a structure $\mathcal{U}$ such that the sentences of $\mathscr{Q}$ can be evaluated in $\mathcal{U}$.

\section{Proposed Formalism}

In order to formally specify $M \& S$, we need to define six basic terms that are commonly, and ambiguously, found in the literature: (1) reference model, (2) modeling question, (3) model, (4) valid model, (5) simulation, and (6) simulator. The definitions of reference model, conceptual model, simulator, and simulation were originally proposed in Ref. [10]. They are reiterated here to provide a unified context for the formal description of the basic tenants of M\&S.

Reference Model: A reference model, denoted $R_{M \& S}$, is a structure $\mathcal{U}[10]$.

A reference model is the universe of interest $A$ and the relationships $R$ between objects in that universe. Model Theory gives us the flexibility of studying several types of relationships within the same universe by changing $R$. In Model Theory $R$ is also known as the interpretation function and it is a concept that is very useful in M\&S because it accounts for the purpose of the model, meaning that a universe can be subject to many interpretations. The $R_{\mathrm{M} \& \mathrm{~S}}$ can be about real or constructed things and captures what we know about problem situations in which these real or imagined things appear. It also captures assumptions/constraints made about the problem situation. Without going into the philosophical aspects of the definition, the $R_{\mathrm{M} \& \mathrm{~S}}$ represents the modeler's worldview of the referent which implies that even for a given universe there is an infinite number of potentially equivalent or competing interpretations.

Modeling Question: A modeling question, denoted $Q_{M \& S}$, is a collection of sentences $\Sigma[10]$.

A modeling question is a collection of sentences to which a truth value needs to be assigned. That assignment is only possible if there exists a reference model that satisfies the $Q_{\mathrm{M} \& \mathrm{~S}}$. In M\&S, the idea of the $Q_{\mathrm{M} \& \mathrm{~S}}$ is essential since modeling is a purposeful abstraction of reality [13]. Consequently, it is this question that bounds what can be asked of a model. We liken the idea of a $Q_{\text {M\&S }}$ to a query. According to Lipski [14], there are two ways to interpret a query in data modeling theory: internal and external. An external interpretation refers to the query directed to the real world. An internal interpretation refers to a query directed to information about the world. Likewise in $M \& S$, a $Q_{\text {M\&S }}$ can be answered by directing the question to a referent or a model of the referent. As a referent in this case is a problem situation, an external interpretation of the $Q_{\mathrm{M} \& \mathrm{~S}}$ is not possible. This means that only an internal interpretation is possible and the $R_{\mathrm{M} \& \mathrm{~S}}$ is that interpretation.

Having formally defined reference model and modeling question, we can define a model in M\&S. It is important to note that in order to avoid confusion we will refer to a model in M\&S as the Renner definition [13], noted $M_{\mathrm{M} \& S}$, in contrast to a model in the Model Theoretic sense.

Model: A model in M\&S, denoted $M_{\mathrm{M} \& \mathrm{~S}}$, is a language 2 [10].

The $M_{\mathrm{M} \& \mathrm{~S}}$ can be generated from a set of elementary sentences so a $M_{\mathrm{M} \& \mathrm{~S}}$ can equivalently be defined 
as the set of elementary sentences and the logical connectors used to generate a language. While a $M_{\mathrm{M} \& \mathrm{~S}}$ is a language or a set of sentences this only captures the syntax of an $M_{\mathrm{M} \& \mathrm{~S}}$ as opposed to its semantics (or interoperation). We are able to recognize sentences that belong to the $M_{\mathrm{M} \& \mathrm{~S}}$ and determine whether an external modeling question is a subset of our $M_{\mathrm{M} \& \mathrm{~S}}$ but we do not address the meaning or interpretation of a modeling question.

It is important to note that the internal modeling question is contained within the $M_{\mathrm{M} \& \mathrm{~S}}$ and we purposefully separate the formulation of the question from its interpretation which we will address later. The notion of a theory is obviously very important in M\&S since we are not only concerned with the existence of $M_{\mathrm{M} \& \mathrm{~S}}$ but we are also in the correct $M_{\mathrm{M} \& \mathrm{~S}}$. In general we call validation the process of ascertaining that the correct model was built, and we say that the $M_{\mathrm{M} \& \mathrm{~S}}$ is valid with respect to the $R_{\mathrm{M} \& \mathrm{~S}}$. This leads us to purposefully distinguish between a model $\left(M_{\mathrm{M} \& \mathrm{~S}}\right)$ and a valid model in order to reflect the relative nature of truth in M\&S models. This distinction is because a $M_{\mathrm{M} \& \mathrm{~S}}$ can be partly or wholly satisfiable with respect to a $R_{\mathrm{M} \& \mathrm{~S}}$. Having introduced the notion of model in M\&S, we can define a valid model.

Valid Model: $A M_{M \& S}$ is valid, denoted $V_{M \& S}$, if and only if it is satisfiable.

To further elaborate on this definition we need to consider two uses cases. The first occurs when one builds a $R_{\mathrm{M} \& \mathrm{~S}}$ then generates a $V_{\mathrm{M} \& \mathrm{~S}}$. The second use case occurs when one builds a $M_{\mathrm{M} \& \mathrm{~S}}$ and attempts to validate it:

1. From a $R_{\mathrm{M} \& \mathrm{~S}}$ to a $V_{\mathrm{M} \& \mathrm{~S}}$ : Departing from a reference model $\mathcal{U}$, it is possible to generate a language 2 . More importantly, we can capture our assumptions in the form of axioms, as part of the $R_{\mathrm{M} \& \mathrm{~S}}$, and generate a $V_{\mathrm{M} \& S}$.

2. From a $M_{\mathrm{M} \& \mathrm{~S}}$ to a $R_{\mathrm{M} \& \mathrm{~S}}$ : Using Model Theory again, a valid model in M\&S ( $\left.V_{\mathrm{M} \& \mathrm{~S}}\right)$ is a collection of sentences that are true under a reference model $\left(R_{\mathrm{M} \& \mathrm{~S}}\right)$.

In order for $M_{\mathrm{M} \& \mathrm{~S}}$ to be simulated by a computer it must be regular which implies that it is context-free. Without discussing the implications of this compromise (the exploration of this implication is left to the reader), we assume that $M_{\mathrm{M} \& \mathrm{~S}}$ is regular and provide the following definitions:

- Simulator: A simulator is a FSM [10].

- Simulation: A simulation is the $2_{\mathrm{FSM}}$ of a $V_{M \& S}[10]$.

A simulator is any machine capable of generating the $M_{\mathrm{M} \& \mathrm{~S}}$ (see definitions) and a simulation is the realization of the FSM of the $V_{\text {M\&s. }}$ It is important to note that the notion of verification is subsumed in this definition since the simulator must always generate the model in order for the simulation to occur, otherwise we consider the simulator as having generated a $M_{\mathrm{M} \& \mathrm{~s}}$.

\section{IMPLICATIONS OF THE MT-MS FORMALISM}

The implications of the proposed formalism go beyond having unambiguous terms defined and providing a lingua franca when talking about M\&S. The formalism is important because we can begin to explain other complex concepts in M\&S, namely, conceptual modeling, validity, and interoperability.

\section{On Conceptual Modeling}

Traditionally, a conceptual model is considered a specification of a model, that is implementation independent. Using the proposed formalism, we propose that the process of conceptual modeling encompasses the specification of a reference model (that contains assumptions/constraints), the specification of modeling questions, and the specification of a $V_{\text {M\&s. }}$. This process leads to the following definition:

Conceptual Model: A conceptual model is a $V_{M \& S \text {. }}$

Consequently, conceptual modeling is the process of specifying a $V_{\mathrm{M} \& \mathrm{~S}}$. A conceptual model is a theory of the reference model $R_{\mathrm{M} \& \mathrm{~S}}$. As such, the conceptual model should not violate any of the assertions made in the reference model $R_{\mathrm{M} \& \mathrm{~S}}$.

Figure 1 presents a framework for conceptual modeling where the problem situation is captured in a structure $\mathcal{U}$ which acts as a $R_{\mathrm{M} \& \mathrm{~s}}$. This framework is adapted from Ref. 10 and has been applied to model the effect of sea level rise on a geographic region. The structure $\mathcal{U}$ represents what we know about a problem situation and can be captured as an ontology. The $Q_{\mathrm{M} \& \mathrm{~S}}$, or sentences $\Sigma$, are what we don't know and want to ask to the $R_{\mathrm{M} \& \mathrm{~S}}$. Pragmatically, sentences $\Sigma$ can be formulated using the Manchester OWL syntax [15]. The $M_{\mathrm{M} \& \mathrm{~S}}$ is a conceptual model that captures how we intend to answer the $Q_{\mathrm{M} \& \mathrm{~S}}$. The $V_{\mathrm{M} \& \mathrm{~S}}$ is the $M_{\mathrm{M} \& \mathrm{~S}}$ that answers the $Q_{\mathrm{M} \& \mathrm{~S}}$ and the result of the conceptual modeling process. It can be captured using the Unified Modeling Language (UML) or the Systems Modeling Language (SysML) $[16,17]$. Finally, the simulation is the finite state machine realization of the conceptual model. Although the simulation is not part of the conceptual modeling process, it is shown in the figure to showcase the process of validation.

Lastly, while traditionally UML and SysML are used to capture information of a referent using natural language in a structured manner, using the proposed formalism would allow for automation using tools that can directly compute a conceptual model and check for validity using a reference model. For instance, model checking algorithms can be used to test a $M_{\mathrm{M} \& \mathrm{~S}}$ against a $R_{\mathrm{M} \& \mathrm{~S}}$ to assert whether or not the $M_{\mathrm{M} \& \mathrm{~S}}$ is a $V_{\mathrm{M} \& \mathrm{~S}}$ [18]. In other words, 


\section{FIGURE 1}

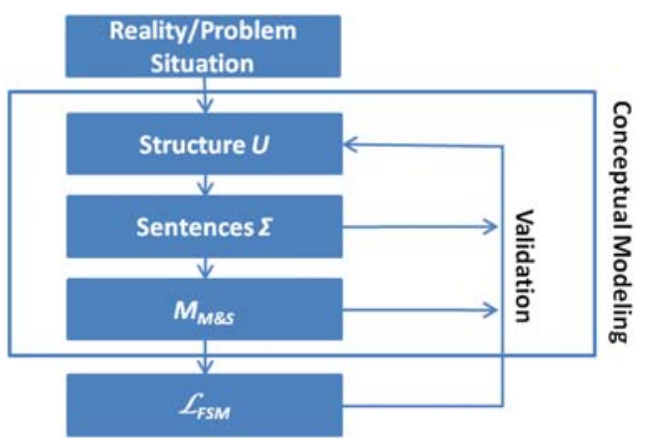

An M\&S framework for conceptual modeling.

one can use software such as Prototype Verification System (PVS) to establish that the assigned truth value to $\Sigma$ sentences is consistent with the $R_{\mathrm{M} \& \mathrm{~S}}$ [19].

\section{On Validity}

Section "Proposed Formalism" provides the definition of a valid model ( $V_{\mathrm{M} \& \mathrm{~S}}$ ). Based on the formalism, validity within problem situations is established through the cor-

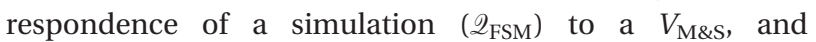
through the correspondence of a $V_{\mathrm{M} \& \mathrm{~S}}$ to a $R_{\mathrm{M} \& \mathrm{~S}}$. In other words, validity in $\mathrm{M} \& \mathrm{~S}$ is the traceability of a simulation up to the reference model when referring to particular modeling questions and it is evaluated through satisfiability. It is noted that an objective measure of validation within problem situations is only possible when comparing model with model. A direct link between simulations and the problem situation (an external interpretation) is possible through subject matter experts.

Axiomatic M\&S Validity: Axiomatic M\&S validity is the correspondence between $\mathscr{2}_{\mathrm{FSM}}, V_{\mathrm{M} \& \mathrm{~S}}$ and a $R_{M \& S}$.

In order to further explain validity, we still need to consider whether the $V_{\mathrm{M} \& \mathrm{~S}}$ is generated from a $R_{\mathrm{M} \& \mathrm{~S}}$ or one builds a $M_{\mathrm{M} \& \mathrm{~S}}$ and attempts to validate it:

- From a $R_{M \& S}$ to a $V_{M \& S}$ : By generating a $V_{\mathrm{M} \& S}$ from a $R_{\mathrm{M} \& \mathrm{~S}}$, we are equating validity with satisfiability as we are stating that validity is a relation between the modeling question $Q_{\mathrm{M} \& \mathrm{~S}}$ and the reference model $R_{\mathrm{M} \& \mathrm{~S}}$ that captures the assumptions and constraints in the form of axioms.

There are two ways to satisfy the modeling question $Q_{\mathrm{M} \& S}$. Either the $Q_{\mathrm{M} \& \mathrm{~S}}$ is a set of axioms or there exists a

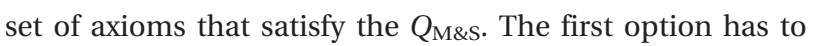
be rejected since we do not want to axiomatically accept the $Q_{\mathrm{M} \& \mathrm{~S}}$. We will further discuss why accepting the first option is counter-productive in the next use case. The sec- ond option implies that we need a set of axioms from which we can evaluate a $Q_{\text {M\&s. }}$. These axioms are the assumptions and constraints that we put on the problem situation. They serve as the basis for establishing what we accept as being true about the referent. The set of axioms bridges the real or imagined world modeled and the $R_{\mathrm{M} \& \mathrm{~S}}$. Consequently, the $R_{\mathrm{M} \& \mathrm{~S}}$ becomes our reality that contains the $Q_{\mathrm{M} \& \mathrm{~S}}$ that it can answer.

- From a $M_{M \& S}$ to a $R_{M \& S}$ : The transition from a $R_{\mathrm{M} \& \mathrm{~S}}$ to a $M_{\mathrm{M} \& \mathrm{~S}}$ is usually a mental process and in practice modelers simply write the theory; this means that we only capture what we refer to as a conceptualization, simplification, or abstraction as a theory and assume that this is sufficient to answer the

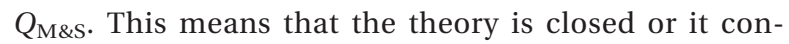
tains all of its consequences (see Section "Proposed Formalism") for the $Q_{\text {M\&s. }}$. This approach is fraught with danger since the assumptions and constraints are implicit. In this case, the model is axiomatically valid and what we call validation as defined in the literature is really a process of ascertaining that the axioms included are sufficient to answer some external modeling question and that the assumptions though not captured are reasonable. This process usually involves subject matter experts and centers around the use of natural language to calibrate the $M_{\mathrm{M} \& \mathrm{~S}}$ in order to reflect what is expected or understood from the referent. This process does not change the definition of validity and the reference in this case model is a mental model shared by the participants.

Based on the previous discussion, the traditional idea of validation as the last activity of a modeling process needs to be reconsidered. By treating the $R_{\mathrm{M} \& \mathrm{~S}}$, the $V_{\mathrm{M} \& \mathrm{~S}}$, the simulator and the simulation as an equivalence class, we are guaranteeing the validation process by definition. In other words, by construction it is meaningless to talk about M\&S unless the model is valid with respect to the $R_{\mathrm{M} \& \mathrm{~S}}$ and there is a simulator that is able to generate a $V_{\mathrm{M} \& \mathrm{~S} \text {. In prac- }}$ tice, it means that from the requirements gathering to formulate a $Q_{\mathrm{M} \& \mathrm{~S}}$, to the conceptual model all the way to the execution, the modeler has to ensure consistency across the board such that every artifact generated is equivalent to the same consistent theory. Furthermore, the resulting simulation combines deductive and inductive processes which implies that empirical efforts should be accompanied with axiomatic validation efforts as conducted in disciplines such as aerospace engineering [1].

\section{On Interoperability}

The observation of equivalence between modeling question, the $V_{\mathrm{M} \& \mathrm{~S}}$, the simulator, and the simulation is very important for interoperability, as interoperability itself 


\section{FIGURE 2}

\begin{tabular}{|l|l|l|}
\hline Term & Traditional M\&S Definition & Model Theory M\&S Definition \\
\hline Model & Abstraction of reality & A language $\mathcal{L}$, denoted $M_{\text {M\&s }}$ \\
\hline Simulation & Execution of a model over time & The $\mathcal{L}_{\text {Mss }}$ of a Valid $M_{\text {Mss }}$ \\
\hline Simulator & A computer & A FSM \\
\hline Modeling Question & A Query & A collection of sentences $\Sigma$ \\
\hline Reference Model & N/A & A structure $\boldsymbol{U}$ \\
\hline Valid Model & $\begin{array}{l}\text { Adequate representation of a } \\
\text { phenomenon }\end{array}$ & A satisfiable $M_{\text {M\&s }}$ \\
\hline
\end{tabular}

Traditional and model theory comparison of terms for M\&S.

must abide by the rules of satisfiability, consistency, and the definitions provided in the previous section. The proposed formalism provides a way of formally defining and explaining interoperability as well. In order to do so, we use Model Theory to formally define data, information, and useful information.

Data: Given a $R_{M \& S}, \quad$ a structure $\mathcal{U}$ defined by $\mathcal{U}:\left\langle A, R>\right.$, data is the universe $A$ of a $R_{M \& S}$.

Information: Given a $M_{M \& S}$, a language $\mathscr{2}$, and $\varphi$ a sentence in $M_{M \& S}$, information is a sentence.

Useful Information: Given a $R_{M \& S}, a M_{M \& S}$, and $\varphi$ a sentence in $M_{M \& S}$, information is useful and it is satisfiable under the $R_{M \& S}$.

By defining data as the universe $A$, we cover all objects that are part of the universe including constants, functions, and relations. In practical terms, the notion of data as a static set of symbols is covered by constants. However, it is important to note that we include functions and relations as well. Their inclusion means that the combination of data and its interpretation has an equivalent finite state machine under certain conditions (see Section "Proposed Formalism"). Further, the definition of data does not include its interpretation which gives us the flexibility of an infinite combination of data under a given interpretation or the ability to generate multiple finite state machines for the same data. This separation allows for a distinction between the exchange of data and the exchange of information such that we can study the differences and similarities between the two.

The transition from data to information logically follows by observing that data under an interpretation (a statement) is information. By defining information as a statement that can be true or false, we bring the flexibility provided by Model Theory into M\&S, namely, the idea of truth being relative. In this case, there are an infinite number of statements about data that can be generated by simply changing the interpretation function and each one of these statements has to be satisfiable under a reference model in order to be deemed useful within that universe.
Previously, we have defined a model as a $M_{\mathrm{M} \& \mathrm{~S}}$ and defined a $V_{\mathrm{M} \& \mathrm{~S}}$ as the theory of that $M_{\mathrm{M} \& \mathrm{~S}}$. We also understand informally that interoperability involves the exchange of information and the ability to use the information. We have defined information as a sentence and information is useful if there is a structure under which it is satisfied. Thus, interoperability can be defined as the exchange of useful information:

Interoperability: Interoperability is the generation of a theory of a reference model.

This is a very general definition for interoperability and has several implications:

- Model Interoperability: Since a $V_{\mathrm{M} \& \mathrm{~S}}$ is a theory of a $R_{\mathrm{M} \& \mathrm{~S}}$, the definition of interoperability implies that a $V_{\mathrm{M} \& \mathrm{~S}}$ is interoperable with a $R_{\mathrm{M} \& \mathrm{~S}}$. Otherwise stated, a $M_{\mathrm{M} \& \mathrm{~S}}$ is interoperable with a $R_{\mathrm{M} \& \mathrm{~S}}$ if and only if it is a $V_{\mathrm{M} \& \mathrm{~S}}$ of that $R_{\mathrm{M} \& \mathrm{~S}}$. In practice, this implies that it is possible to build an interoperable $M_{\mathrm{M} \& \mathrm{~S}}$ by simply ensuring that the $M_{\mathrm{M} \& \mathrm{~S}}$ is valid with respect to a $R_{\mathrm{M} \& \mathrm{~S}}$. It also implies that contrary to the intuitive belief that $M_{\mathrm{M} \& \mathrm{~S}}$ should be built to answer very specific questions, interoperable $M_{M \& S}$ should be built to answer broad modeling questions from which multiple sub-questions can be derived. For instance, instead of making two specific $M_{\mathrm{M} \& \mathrm{~S}}$ interoperable through model specific interfaces, it is preferable to make $M_{\mathrm{M} \& \mathrm{~S}}$ interoperable by aligning their axiomatic structures and generating a consistent set of axioms across the $M_{\mathrm{M} \& \mathrm{~S}}$. Thus, interoperability becomes the validation of two or more $M_{\mathrm{M} \& \mathrm{~S}}$ with respect to some $R_{\mathrm{M} \& \mathrm{~s}}$.

- Simulation Interoperability: Since interoperability is the generation of a theory of a $R_{\mathrm{M} \& \mathrm{~S}}$ and simulation is the FSM realization of a $V_{\mathrm{M} \& \mathrm{~S}}$, simulation interoperability is simply the generation of a $V_{\mathrm{M} \& \mathrm{~S}}$ from one or more simulators (FSM languages). In practice, the focus is mostly on making two or more simulations interoperable. Under this definition this 
is only possible if the $M_{\mathrm{M} \& \mathrm{~S}}$ formed by putting the simulations together is valid.

These two observations are counter-intuitive at first sight and therefore deserve more explanation. A model is defined as a language $\mathscr{2}$ and in keeping with the nature of Model Theory, a model is not right or wrong (or even valid or invalid). The determination of validity is always with respect to that which is modeled. Consequently, a model becomes a theory when there is a reference model that it purports to answer. In our definitions, we call the process of generating such a theory interoperability. This means however that we have equated interoperability with validity by defining validity as a special form of interoperability. Namely, the interoperability of the $M_{\mathrm{M} \& \mathrm{~S}}$ with the $R_{\mathrm{M} \& \mathrm{~S}}$. This is important because it means that at a minimum every $V_{\mathrm{M} \& \mathrm{~S}}$ is interoperable. It also means that a $M_{\mathrm{M} \& \mathrm{~S}}$ can generate multiple $V_{\text {M\&s. }}$. We can also define and distinguish reuse from interoperability.

Reuse: Reuse is the number of theories that can be generated from a language.

Equivalently reuse is the number of models of a theory. By either definition, reuse is a function of the modeling question which fits the intuitive understanding of using an M\&S model for multiple purposes. It is important to note that a theory is not reusable; rather a theory is one of the possible uses of a language for a given modeling question. In practice, it means that it is impossible to reuse a valid implementation of a model. However, it is always possible to reuse the model to generate a theory that can answer other modeling questions.

\section{concLusIONS}

In order to advance $M \& S$ as a scientific endeavor, we need to formally specify terms in order to eliminate ambiguity in their use. In order to do so, we use Model Theory as the means to formalize M\&S. Figure 2 presents M\&S commonly used terms in an informal way compared with the proposed Model Theory-based definitions. It is noted that the proposed definitions are not formal for formality's sake. Instead their formality provides a platform for structured discussion and they allowed us to unambiguously explain ambiguous terms. Furthermore, the provided definitions provide a platform for studying areas of importance to $M \& S$ such as validity, interoperability, and conceptual modeling.

The formalism also provides a pragmatic advantage by enabling us to semi-automate the validation and interoperation processes. Currently, these processes are conducted in an ad hoc manner opening them to accept inconsistencies while being labor intensive. As validity is subsumed under interoperability, we can use model checking tools to test that a $M_{\mathrm{M} \& \mathrm{~S}}$ is satisfiable under a reference model. In other words, we assert whether or not the $M_{\mathrm{M} \& \mathrm{~S}}$ is a $V_{\mathrm{M} \& \mathrm{~S}}$.

Finally, the formalism also allows us to establish that all $M_{\mathrm{M} \& \mathrm{~S}}$ satisfiable under the same reference model are composable and their respective simulations are interoperable. This facilitates the reuse of simulations as they don't have to be checked against one another, but against a reference model. In future work, we look to continue to realize these definitions and their constrains in a framework that facilitates the development of valid, interoperable, and reusable models through conceptual modeling.

\section{REFERENCES}

1. Zeigler, B.P.; Praehofer, H.; Kim, T.G. Theory of Modeling and Simulation: Integrating Discrete Event and Continuous Complex Dynamic Systems. Academic press, San Diego, CA, 2000. Int. J. Robust Nonlinear Control, 12: 91-92. doi: 10.1002/ rnc.610.

2. Petty, M.D.; Weisel, E.W. A composability lexicon. In Proceedings of the Spring 2003 Simulation Interoperability Workshop, Orlando, FL, 2003; pp 181-187.

3. Boschetti, F; McDonald, D.; Gray, R. Complexity of a modelling exercise: A discussion of the role of computer simulation in complex system science. Complexity 2008, 13, 21-28.

4. Vennix, J.A.M.. Group Model Building. Wiley: New York, 1996.

5. Reynolds, P.F. 2008. The Role of Modeling and Simulation, in Principles of Modeling and Simulation: A Multidisciplinary Approach (eds J. A. Sokolowski and C. M. Banks), John Wiley \& Sons, Inc., Hoboken, NJ, USA. doi: 10.1002/ 9780470403563.ch2.

6. Axelrod, R. Advancing the art of simulation in the social sciences. Complexity 1997, 3, 16-22.

7. Epstein, J.M. Agent-Based Computational Models and Generative Social Science. Complexity 1999, 4, 41-60.

8. Maldonado, C.E.; Cruz, G.; Nelson, A. The complexification of engineering. Complexity 2012, 17, 8-15.

9. Robinson, S. Simulation: The Practice of Model Development and Use. Wiley, Chichester, UK, 2004.

10. Tolk, A.; Diallo, S.Y.; Padilla, J.J.; Herencia-Zapana, H. Reference modelling in support of M\&S-foundations and applications. Journal of Simulation 2013, 7, 69-82.

11. Anne, Geraci. 1991. IEEE Standard Computer Dictionary: Compilation of IEEE Standard Computer Glossaries. IEEE Press, Piscataway, NJ, USA.

12. Weiss, W.; D’Mello, C. 1997. Fundamentals of Model Theory. Department of Mathematics, University of Toronto, Canada. Accessed May 1. http://www.math.toronto.edu/weiss/model_theory.pdf.

13. Renner, S. A community of interest approach to data interoperability. In Federal Database Colloquium, San Diego, CA, Vol. 1,$2001 ; \mathrm{p} 2$.

14. Lipski, W., Jr. On semantic issues connected with incomplete information databases. ACM Trans Database Systems (TODS) 1979, 4, 262-296. 
15. Horridge, M.; Drummond, N.; Goodwin, J.; Rector, A.; Stevens, R.; Wang, H.H. The Manchester OWL syntax. 2006. OWL: Experiences and Directions (OWLED 06) Athens, Georgia: pp 10-11.

16. Friedenthal, S.; Moore, A.; Steiner, R. A Practical Guide to SysML: The Systems Modeling Language. Morgan Kaufmann, San Francisco, CA, 2011.

17. Rumbaugh, J.; Jacobson, I.; Booch, G. Unified Modeling Language Reference Manual. The Pearson Higher Education, 2004.

18. Clarke, E.M.; Grumberg, O.; Peled, D. Model Checking. The MIT press, Cambridge, MA, USA, 1999.

19. Owre, S.; Rushby, J.M.; Shankar, N. Pvs: A Prototype Verification System. In Automated DeductionCADE-11, Springer, Saratoga, NY, 1992; pp 748-752. 